\title{
5 Research Square

\section{Visual outcome after trifocal IOL implant in a patient with cornea guttata. A case report.}

Jorge E.Valdez-Garcia ( $\sim$ jorge.valdez@itesm.mx )

Gustavo Ortiz-Morales

Instituto Tecnologico y de Estudios Superiores de Monterrey

Nallely Morales-Mancillas

Hospital Zambrano Hellion

Denise Loya-Garcia

Hospital Zambrano Hellion

Julio Hernández-Camarena

Hospital Zambrano Hellion

\section{Case report}

Keywords: Corneal endothelium, cornea guttata, Fuchs' endothelial dystrophy, cataract surgery- premium IOL.

Posted Date: March 26th, 2019

DOI: https://doi.org/10.21203/rs.2.498/v1

License: (c) (1) This work is licensed under a Creative Commons Attribution 4.0 International License.

Read Full License 


\section{Abstract}

Background We report the case and management of a patient that started with primary cornea guttata symptoms after cataract surgery with trifocal intraocular lens (IOL) implant. The unusual postoperative presentation of the disease in association with a trifocal IOL resulted in increased corneal surface aberrations that resulted in visual dissatisfaction. Case presentation A 67 year-old male was evaluated due to blurred vision for two months, as well as a desire to stop using glasses in his everyday life. At the initial refraction, myopia, corneal astigmatism and nuclear cataract were diagnosed in both eyes. $\mathrm{A}$ nuclear cataract was diagnosed in both eyes. Phacoemulsification surgery was performed in OS, implanting a trifocal IOL. The postoperative refraction in OS was $-1.25 \mathrm{DC} \times 120^{\circ}$, with a best-corrected visual acuity (BCVA) of 20/20. Six months later, the patient kept complaining of blurred vision and night glare. In slit lamp examination, corneal guttae were denoted and changes in corneal endothelium morphology suggested primary cornea guttata. A monofocal IOL exchange was performed in order to reduce corneal aberrations with excellent outcome at 2-year follow up. Conclusions Screening for endothelial dysfunction is a key element for successful outcomes in cataract surgery. Corneal guttae accent optical aberrations when in combination with premium IOLs.

\section{Background}

Corneal guttae are focal excrescences of extracellular matrix in Descemet's membrane. They initially appear on specular reflection as scattered and isolated dark structures. ${ }^{1}$ Specular microscopy confirms the clinical findings. ${ }^{2}$

Because symptoms start around the fifth decade of life, a significant number of patients with cornea guttata could have cataracts as well. Likewise, symptoms caused by guttae are similar to those caused by cataracts (glare, loss of contrast sensitivity, blurred vision). ${ }^{3}$ The age overlap between this two conditions poses a challenge for the ophthalmologist in the surgical decision making, as endothelial cell damage during surgery may accelerate endothelial cell loss between $4-25 \%{ }^{3}$

\section{Case Presentation}

A 67 year-old male, of Jewish descent, came for an ophthalmological evaluation after a two-month history of blurry vision, as well as a desire to stop using eyeglasses in his everyday life. He does not refer any other relevant medical history.

At his initial assessment, uncorrected distance visual acuity (UDVA) was 20/20 in OD and 20/30 in OS. Uncorrected near visual acuity was $\mathrm{J} 3$. The initial refraction was $-0.50 \mathrm{DC} \times 120^{\circ}$ in $\mathrm{OD}$ and $-1.75 \mathrm{DS} /-1.50 \mathrm{DC} \times 70^{\circ}$ in OS. Corrected distance visual acuity (CDVA) was 20/20 for both eyes. Nuclear cataracts were observed in both eyes during slit lamp examination. The other anterior segment structures and fundus examination were unremarkable. The assessment for a surgical solution was initiated. 
A corneal topography using the OPD Scan-III (Nidek Co., LTD. Gamagori, Japan) was performed in OS. SimK was 42.22@135\% /41.45@45․ For IOL power calculation, an optical biometry using IOLMaster 500 (Carl Zeiss, Oberkochen, Germany) together with Barrett Universal II formula was used. The average calculation of lens power was +17.00D for OS.

After assessment, phacoemulsification surgery and IOL placement were done in OS, implanting a trifocal lens model AT LISA tri 839MP (Carl Zeiss, Oberkochen, Germany). The surgery was performed uneventfully.

During the first week of the postoperative period, the UDVA was $20 / 20$ in OS. The refraction at this time was $-1.25 \mathrm{DC} \times 120^{\circ}$.

Six months into the postoperative period, the patient noted persistent blurred vision and night glare appeared. Slit lamp examination was performed and cornea guttata was observed. A specular microscopy (Fig 1) with EM-3000 (Tomey, Phoenix, AZ, USA) and a corneal topography using OPD ScanIII were performed.

In OD, the specular microscopy (central) showed a central corneal thickness (CCT) of 535, cell density (CD) of 1716 cells $/ \mathrm{mm} 2$, polymegathism of $53 \%$ and pleomorphism of $42 \%$. In OS, the examination showed a CCT of 539, CD of 934 cells/mm2, polymegathism of $113 \%$ and pleomorphism of $24 \%$. Both eyes had presence of guttae.

In OD, the OPD scan showed a SimK of 42.03@65\%/41.82@155. The Zernike analysis showed a total of 0.660, with 0.469 @139 of tilt. Meanwhile, in OS, SimK was 42.08@15\%/41.56@105 and the Zernike analysis showed a total of 1.396 , with $0.539 @ 295^{\circ}$ of tilt and 0.410 of high order aberrations (HOAs). A well-centered IOL was observed.

As visual symptoms didn't improve over time, or with the spherocylindrical correction of residual error, a surgical option was preferred, and an IOL exchange was proposed. A second low-frequency optic interferometry with IOL Master 500 was performed, and using Barrett Universal II Formula, an average lens power of +18.00D was calculated. A monofocal aspheric IOL, the ZCB00 (Abbott Medical Optics Inc, Santa Ana, CA, USA), was favored.

During the first week after the IOL exchange, UDVA was 20/25. Refraction at this time was $-0.50 \mathrm{Ds} /-0.50 \mathrm{DC} \times 90^{\circ}$ in OS. Postoperative period developed without complications.

Patient follow-up was given for two years. His last UDVA was 20/20 in both eyes and near visual acuity was J1. His last specular microscopy showed a CCT of 593, CD of 1332 cells $/ \mathrm{mm} 2$, polymegathism of $64 \%$ and pleomorphism of $33 \%$ in OD; and a CCT of 546, CD of 1105 cells $/ \mathrm{mm} 2$, polymegathism of $68 \%$ and pleomorphism of $26 \%$ in OS.

\section{Discussion}


The growing requirements of adults over 65 years old and the increasing popularity of premium IOLs, warrant a meticulous preoperative screening. Although cataract surgery outcomes and complications have remained stable for the last decade, preoperative ophthalmological evaluation has not been standardized. ${ }^{4,5}$

IOL options must be carefully explored when corneal guttae are present. Wacker et al. ${ }^{6}$ found that anterior corneal HOAs were increased in moderate and advanced Fuchs' endothelial dystrophy (FED), compared with controls, before clinically visible corneal edema. Likewise, posterior corneal HOAs and corneal backscatter were increased even in mild FED, suggesting that surface aberrations may occur earlier than previously thought. Corneal guttae reduce contrast sensitivity and by implanting premium $\mathrm{IOL}$, a second source of optical degradation is added. ${ }^{7,8}$ Corneal surface aberrations, in combination with premium IOLs, can result in high visual dissatisfaction. Photopic phenomena (38.2\%) and blurred vision $(36.8 \%)$ are the most common presenting symptoms. ${ }^{9}$

A study by Watanabe et al. ${ }^{8}$ concluded that straylight from corneal guttae may be the main cause of optical degradation. Quantification of corneal guttae may offer a method for objectively evaluating quality of vision in patients with FED, even those who have cataract.

In order to improve our patient's visual quality, IOL options were explored. Monofocal IOL exchange was the optimum choice in our patient. Tecnis ZCB00 was selected for its association with reduced chromatic aberration. Tecnis IOLs made of the acrylic Sensar material were not associated with glistenings except for only one in-vitro study that found minimal non-significant glistenings. ${ }^{10}$

\section{Conclusions}

Screening for endothelial dysfunction is a key element for successful outcomes in cataract surgery. We recommend screening for endothelial pathology in all patients who are candidates to cataract surgery, especially when considering premium IOLs, as this kind of IOL may magnify HOAs and could add a second layer of optical degradation in patients with corneal guttae.

An IOL exchange alone was proposed as a final surgical solution. Slow progress and improvement of visual quality was observed in the follow-up at 2 years.

\section{Declarations}

\section{Ethics approval and consent to participate}

*This study was approved by the Ethics Committee of the Tecnologico de Monterrey School of Medicine (Monterrey, Mexico) and followed the tenets of the Declaration of Helsinki and its later amendments. Informed consent was obtained for the participating subject. 


\section{Consent for publication}

*The patient gave written consent for publication.

\section{Availability of data and material}

*Data sharing is not applicable to this article as no datasets were generated or analyzed during the current study.

\section{Competing interests}

*The authors declare that they have no conflict of interest or financial disclosures.

\section{Funding}

*No funding was obtained for the design, collection of data, analysis or writing of this manuscript.

\section{Authors' contributions}

JEVG: Concept and design, data analysis/interpretation, critical revision of manuscript, supervision, final approval.

GOM: Data acquisition, data analysis/interpretation, drafting manuscript, final approval.

NMM: Data acquisition, data analysis/interpretation, drafting manuscript, final approval.

DLG: Concept and design, data analysis/interpretation, critical revision of manuscript, supervision, final approval.

JHC: Concept and design, data analysis/interpretation, critical revision of manuscript, supervision, final approval.

All authors read and authorized the final version of the manuscript.

\section{Acknowledgements:}

None 


\section{References}

1.Laing RA, Leibowitz HM, Oak SS, et al: Endothelial mosaic in Fuchs' dystrophy. Arch Ophthalmol 1981; 99: pp. 80-83

2.Kitagawa K, Fujisawa A, Mizuno T, \& Sasaki K (2001). Twenty-three cases of primary cornea guttata. Japanese journal of ophthalmology, 45(1), 93-98.

3.Cleynenbreugel HV, Remeijer L, \& Hillenaar T (2014). Cataract Surgery in Patients with Fuchs Endothelial Corneal Dystrophy. Ophthalmology,121(2), 445-453. doi:10.1016/j.ophtha.2013.09.047

4.Date RC, Al-Mohtaseb ZN (2017). Advances in Preoperative Testing for Cataract Surgery. International Ophthalmology Clinics,57(3), 99-114. doi:10.1097/iio.0000000000000171

5.Day AC, Donachie PH, Sparrow JM, \& Johnston RL (2015). The Royal College of Ophthalmologists' National Ophthalmology Database study of cataract surgery: Report 1, visual outcomes and complications. Eye,29(4), 552-560. doi:10.1038/eye.2015.3

6.Wacker K, Mclaren JW, Amin SR Baratz KH, \& Patel S (2015). Corneal High-Order Aberrations and Backscatter in Fuchs Endothelial Corneal Dystrophy. Ophthalmology,122(8), 1645-1652. doi:10.1016/j.ophtha.2015.05.005

7.Lichtinger A, Yeung SN, \& Kim T (2012). Top 5 Pearls to Consider When Implanting Advancedtechnology IOLs in Patients With a Compromised Cornea. International ophthalmology clinics, 52(2), 5963.

8. Watanabe S, Oie Y, Fujimoto H, Soma T, Koh S, Tsujikawa, M, ... Nishida K. (2015). Relationship between corneal guttae and quality of vision in patients with mild Fuchs' endothelial corneal dystrophy. Ophthalmology, 122(10), 2103-2109.

9.Vries NE, Webers CA, Touwslager WR, Bauer NJ, Brabander JD, Berendschot, TT, \& Nuijts RM. (2011). Dissatisfaction after implantation of multifocal intraocular lenses. Journal of Cataract \& Refractive Surgery,37(5), 859-865. doi:10.1016/j.jcrs.2010.11.032

10.Gregori NZ, Spencer TS, Mamalis N, \& Olson RJ (2002). In vitro comparison of glistening formation among hydrophobic acrylic intraocular lenses. Journal of Cataract \& Refractive Surgery, 28(7), 12621268.

\section{Figures}




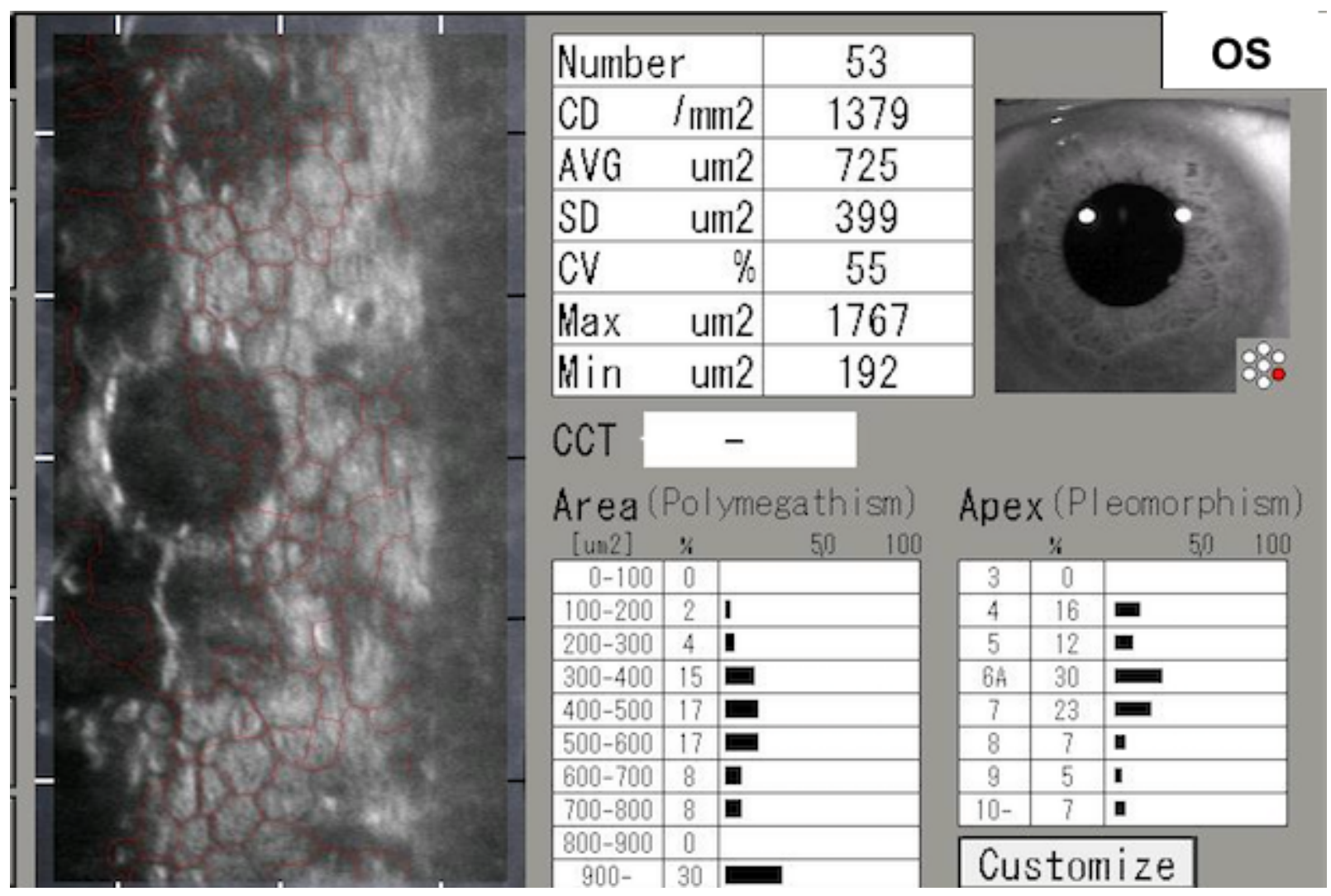

Figure 1

Specular microscopy after trifocal IOL implant shows corneal guttae.

\section{Supplementary Files}

This is a list of supplementary files associated with this preprint. Click to download.

- supplement1.pdf 